\title{
Estabilidad emocional y cortisol como factores diferenciadores de la fibromialgia
}

\author{
Díaz Robredo LA. ${ }^{1}$, Robles Sánchez JI. ${ }^{2}$
}

Sanid. mil. 2011; 67 (1): 11-17; ISSN: 1887-8571

\begin{abstract}
RESUMEN
Este estudio busca un acercamiento al diagnóstico de la fibromialgia desde un modelo psicofisiológico del estrés. En la Introducción justificaremos los principios teóricos en que se basa este trabajo. Las hipótesis planteadas intentarán buscar la diferencia de factores psicológicos y de cortisol entre los grupos control y experimental, así como la correlación entre dichos factores. En el apartado Materiales y métodos nos centramos en los registros de cortisol y de variables de personalidad que realizamos en un grupo de 35 mujeres, 18 con fibromialgia y otras 17 libres de la enfermedad. Los aspectos psicológicos serán recogidos por el test de Personalidad CEP de Pinillos, mientras que el cortisol será medido mediante recolección de saliva. Los resultados, muestran diferencias significativas en los factores psicológicos y fisiológicos de los grupos. En las conclusiones afirmamos que el control emocional y los niveles de cortisol son factores distintivos de esta enfermedad con respecto a un grupo control. Sin embargo, no encontramos una correlación significativa entre control emocional y cortisol pudiendo ser debida a variables extrañas o al escaso número de sujetos de la muestra. Además, los resultados de cortisol suponen una contradicción a estudios previos en los que se observa una reducción de esta hormona en pacientes con fibromialgia, por lo que se recomienda una recogida de cortisol del ciclo diurno para complementar el estudio y solucionar estas disensiones.
\end{abstract}

PALABRAS CLAVE: Fibromialgia, estrés, cortisol, control emocional.

\section{Emotional stability and cortisol as differentiating factors for fibromyalgia SUMMARY}

This study looks for an approach to the diagnosis of fibromyalgia from a psychophysiological model of stress. In the Introduction chapter, we will justify the theoretical principles in which this work is based on. The hypothesis that we suggest will try to find the difference in psychological factors and cortisol between control and experimental group, as well as the correlation of these two factors. In the instrument and method chapter, we will focus on the cortisol and personality variables were achieved in a group of 35 women, 18 with fibromyalgia and 17 disease free. Pinillos CEP Personality test will collect psychological aspects, whereas salivary cortisol will reflect the hormonal level. The results show differences in emotional control and cortisol levels, so in the conclusions chapter, we affirm that these two factors are specific of this disease with respect to a control group. However, there is not a significant correlation between emotional control and cortisol probably due to extraneous variables or the small number of subjects in the sample. Moreover, the results of cortisol are a contradiction to previous studies in which there was a reduction of this hormone in patients with fibromyalgia, so it is recommended a collection of the diurnal cycle of cortisol to complement the study and solve this disagreement.

KEY WORDS: Fibromyalgia, stress, cortisol, emotional control

\section{INTRODUCCIÓN}

Desde los primeros estudios sobre fibromialgia de Smythe ${ }^{1}$ hasta el momento presente, las investigaciones sobre este problema han intentado estudiar la enfermedad desde varios paradigmas. La complejidad no solo de su diagnóstico, sino incluso de la comprensión de su etiología convierten a la fibromialgia en un auténtico reto. Y la enfermedad está de plena actualidad, pues según investigaciones recientes el problema afecta a un $2 \%$ de la población mundial $(3,4 \%$ en mujeres y $0,5 \%$ en hombres $)^{2}$.

El diagnóstico de la fibromialgia (FM) actual ${ }^{3}$, no es sino un conjunto de síntomas físicos consistentes, principalmente, en dolo-

${ }^{1}$ Alf. Psicólogo Reservista Voluntario. Agrupación Base de la Base Aérea de Zaragoza. España.

${ }^{2}$ Cte. Psicólogo. Escuela Militar de Sanidad. Departamento de Psicología. Madrid. España.

Dirección para correspondencia: 1uixdiaz@hotmail.com

Recibido: 12 de enero de 2010

Aceptado: 30 de junio de 2010 res generalizados de tipo no articular y una elevada sensibilidad al dolor en diversos lugares del cuerpo (puntos gatillo), con al menos una duración de 3 meses. Esta definición tan escasa y abierta provoca que el médico, ante la perplejidad que produce la enfermedad, se encuentre muy limitado a la hora de buscar soluciones y explicaciones para el paciente.

El presente estudio es un intento de aportar claves que ayuden a entender la enfermedad en su conjunto, desde un modelo basado en los mecanismos subyacentes del estrés que, es uno de los principales responsables de los problemas de dolor crónico.

\section{Las enfermedades psicosomáticas}

La FM ha sido relacionada con un grupo de alteraciones que hoy día no cuentan con una explicación médica. Son llamadas de varias formas: Enfermedades Psicosomáticas, Síndromes Somáticos Funcionales o Síndromes de Sensibilización Central y comprenden enfermedades como el Síndrome de la Fatiga Crónica, o el Síndrome 
de Intestino Irritable, entre otros. La similitud en las dolencias que provocan (alteraciones del sueño, dolores generalizados, estados de ánimo inestables) llevan a sospechar que pertenezcan a una misma enfermedad. Varios estudios ${ }^{4,5}$ han ido dirigidos a esclarecer la naturaleza de estos problemas. Pese a las similitudes en las formas, los autores no han conseguido demostrar una relación causal entre estas enfermedades. Tan solo han señalado el solapamiento de unos síntomas comunes a todas ellas.

Desde la Medicina Psicosomática, varios estudios sugieren que los Síndromes Funcionales no son tanto un trastorno mental establecido, sino que existe un condicionamiento físico en el día a día, y que los mecanismos relacionados con el estrés pueden ser una fuente importante para entender este tipo de enfermedades ${ }^{6}$.

\section{Estrés y dolor crónico}

La relación entre estrés y dolor es bien conocida desde hace tiempo. Selye ya señalaba en su Síndrome General de Adaptación ${ }^{7}$, la relación entre el cortisol y los procesos de estrés. Aunque los estudios posteriores rebaten la teoría, hoy se continúa asumiendo la importancia de esta hormona en el funcionamiento del eje Hipotálamo-Hipofisario-Adrenal (H-H-A), modelo en el que se basan actualmente la mayoría de las teorías psicobiológicas.

La causa del interés por el eje $\mathrm{H}-\mathrm{H}-\mathrm{A}$ reside en que este es responsable de muchas de las respuestas autónomas del sistema nervioso. Dicho sistema mantiene funciones vitales muy importantes para la supervivencia del individuo y forma parte de un equilibrio interior que ha sido nombrado de varias formas a lo largo de la historia: el «medio interno», de Bernard (1865), la «homeostasis» de Cannon (1932), el «Síndrome General de Adaptación» del ya citado Selye o el más reciente concepto de «alostasis», de McEwen y Wingfield, (2003). Además, se ha comprobado que este sistema se mantiene en estrecha relación con la psicología del individuo y con las reacciones conductuales relacionadas con estrés y dolor.

Para el correcto funcionamiento del equilibrio del sistema nervioso autónomo (SNA) los subsistemas simpático y parasimpático se coordinan entre sí para dar la respuesta adecuada a la situación, activándose uno e inhibiéndose el otro. El sistema simpático desencadena una serie de cambios fisiológicos que buscan la movilización de reservas energéticas y que preparan al individuo para acciones de las denominadas de «lucha o huida», mientras que el parasimpático, de forma antagónica, es el responsable de los procesos de relajación y de vuelta a niveles previos a la situación estresora.

La rama simpática se basa en un doble mecanismo: el más rápido libera noradrenalina y receptores adrenérgicos en los diversos órganos inervados desencadenando reacciones como, por ejemplo: el aumento de la frecuencia cardíaca, la frecuencia respiratoria o el la glucosa en sangre. A su vez, un segundo mecanismo -el llamado eje H-H-A- busca la activación de las glándulas suprarrenales, mediante la liberación de catecolaminas (adrenalina y noradrenalina) en el flujo sanguíneo, posibilitando así la activación general del organismo aun en zonas de escasa o nula inervación. Precisamente, el producto de la activación de las glándulas corticoides es la hormona cortisol.

La activación o estrés puntual produce un aumento de la secreción de glucocorticoides, tanto en cantidad como en frecuencia, volviendo a niveles habituales una vez que el origen del estrés bien ha desaparecido, bien ha dejado de producir la alarma (el organismo se ha adaptado). Sin embargo, en aquellos casos en que los que el individuo no se adapta o no logra recuperar el equilibrio de su medio interno, se puede debilitar la capacidad de autorregulación del eje H-H-A y entrar en un ciclo continuo de producción de cortisol y agotamiento, también llamado estrés crónico.

La influencia del estrés en el dolor se ha investigado de varias formas: el estrés como un amplificador del dolor ${ }^{8}$, el estrés como un factor mantenedor de roles que a su vez producen dolor ${ }^{9} \mathrm{o}$ el estrés como una fuente de alteraciones en sí misma que produce unas consecuencias físicas y psicológicas ${ }^{10}$.

De entre estas consecuencias biopsicológicas, se ha estudiado la alteración de diversas hormonas como catecolaminas, ACTH, hormona de crecimiento y prolactina.

Algunos estudios señalan que ciertas enfermedades relacionadas con el dolor crónico podrían deberse a una acumulación de la hormona cortisol ${ }^{11}$.

\section{Teorías psicológicas de la FM}

La ansiedad y la depresión son compañeros habituales de la FM. En este sentido, numerosos autores han relacionado esta enfermedad con alteraciones psiquiátricas. En uno de los primeros estudios, los autores identifican que hasta el $26 \%$ de los sujetos con FM sufren depresión mayor, frente a un grupo de individuos reumáticos que no reflejan un solo caso. También señala otras diferencias estadísticamente significativas en aspectos como ansiedad vital (26\% del grupo con $\mathrm{FM}$ ), alteraciones por somatización (6\%), bulimia nerviosa (3\%) o personalidad antisocial (1\%) en contraposición a la ausencia de un solo caso del grupo reumático ${ }^{12}$.

Otros autores respaldan estas mayores tasas de alteraciones psicopatológicas en pacientes con FM con la depresión y ansiedad como más frecuentes ${ }^{13,14}$.

Sin embargo, pese a que la relación entre ansiedad, depresión y FM es aceptada como habitual, no está claro aún el peso de estas alteraciones psíquicas en la etiología e incluso en el mantenimiento de la enfermedad. Un estudio reciente realizado con población española, reconoce que la depresión y la ansiedad no son la causa de la FM - de nuevo se trataría más bien de una relación de comorbilidad- y encuentra un perfil específico de la personalidad de estos afectados, que consiste en un nivel muy alto de ansiedad, de miedo al dolor y de hipervigilancia en torno a su propio cuerpo ${ }^{15}$.

En esta misma dirección, otros autores han señalado también la importancia de los factores psicológicos en el tratamiento del dolor crónico como es la $\mathrm{FM}^{16}$.

En la definición de un perfil psicológico que explique la FM, encontramos un factor que relaciona esos rasgos de la personalidad con las respuestas físicas de estrés que puede experimentar el individuo.

La teoría de la personalidad de Eysenck clasifica a los individuos según la duración de la activación del Sistema Nervioso, a partir del concepto de arousal -Teoría del Arousal/Activación ${ }^{17}$ - como estado asociado a la alerta cortical,

Dentro de estas categorizaciones de la Personalidad, el autor define la dimensión Neuroticismo o Inestabilidad Emocional como una disposición temperamental de los individuos hacia las respuestas ansiosas o de estrés. La labilidad emocional es un concepto importante en esta teoría de la personalidad, pues produce una rápida y fácil activación del SNA la cual, a su vez, creará o modificará 
conductas. Los niveles de Extraversión, según Eysenck, correlacionarán inversamente a los niveles de activación del individuo, siendo los introvertidos quienes mayor nivel de activación crónica experimentarán.

Estos conceptos, sobretodo los de inestabilidad emocional y labilidad, son los más relacionados con los factores psicológicos que se medirán en el presente experimento. En concreto, la variable Control Emocional reflejará este aspecto del individuo, indicando la reactividad personal de los individuos hacia los estímulos estresantes.

\section{El eje H-H-A y la fisiología de la FM}

Los estudios que se enumeran a continuación intentan explicar la FM desde un modelo de disfunción del eje H-H-A en el que el fallo de los sistemas homeostáticos debido a situaciones de estrés permanente puedan desembocar en un dolor crónico sin aparentes causas orgánicas.

Desde los primeros estudios realizados en torno al eje H-H-A y la FM, ya se encontraron evidencias de esta relación ${ }^{18-20}$.

En las numerosas investigaciones longitudinales realizadas hasta la fecha se encuentran resultados diversos y, en ocasiones, contradictorios en cuanto a las diferencias en las tasas de cortisol en pacientes con FM respecto a grupos de sujetos sanos, aunque la mayoría de ellos reflejan valores de cortisol inferiores en los pacientes con FM por la mañana y superiores al grupo control por la tarde $\mathrm{t}^{21-25}$.

Se han estudiado también la alteración de otras hormonas en la enfermedad de FM, como hormona del crecimiento, o prolactina sin llegar a resultados concluyentes ${ }^{26,27}$.

En cualquier caso, y pese a que la implicación de los mecanismos hormonales en la FM no está del todo clara, la aparente correlación de la FM y disfunción del eje $\mathrm{H}-\mathrm{H}-\mathrm{A}$ es un hecho admitido por la mayoría de investigadores, tal y como se ha visto en varios de los estudios citados.

Por ello, el presente estudio intentará ahondar un poco más en la investigación del eje H-H-A mediante el análisis de las tasas de cortisol basal en grupos con FM frente a un grupo control libre de esta dolencia y comparar dichos niveles con las variables psicológicas encontradas en los sujetos.

\section{Hipótesis de este estudio}

Justificadas las bases fisiológicas y psicológicas en que se basa dicho experimento, las hipótesis que se intentarán contrastar son las siguientes:

PRIMERA. Que las personas con FM (variable independiente) generan o mantienen perfiles psicológicos específicos y diferentes al grupo control. En concreto, la variable control emocional (variable dependiente) determinará el grado de activación del individuo que se espera sea muy superior en el grupo experimental.

SEGUNDA. Que las personas con FM muestran niveles de cortisol superiores (variable dependiente) al grupo experimental como consecuencia de ese grado de activación.

TERCERA. Que los niveles de cortisol elevados correlacionan positivamente con rasgos de personalidad de bajo control emocional.

\section{MATERIALES Y MÉTODOS}

\section{Participantes}

Cuarenta y dos mujeres distribuidas en dos grupos. El grupo experimental formado por 24 mujeres diagnosticadas de FM y pertenecientes a la Asociación Alavesa de FM (ASAFIMA). El grupo control estaba constituido por 18 mujeres libres de esta enfermedad y que mostraron voluntariamente su interés por participar.

Los criterios de exclusión eran los siguientes: vigilia en las 72 horas previas al experimento por causas ajenas a la FM, consumo de medicación con corticoides, embarazo, alteraciones hormonales, edades inferiores a 40 años o superiores a 60 . El requisito para ser incluido en el grupo experimental era haber recibido el diagnóstico de FM por parte de un médico.

Del grupo experimental inicial de 24 sujetos, se excluyeron a una persona por tomar medicación con corticoides y a 5 por sufrir alteraciones de tiroides y/o estar medicadas al respecto, resultando en un número final de 18 sujetos con FM.

Del grupo control se excluyó a una persona por haber pasado la noche anterior en vigilia por problemas físicos, resultando en 17 sujetos.

\section{Procedimiento}

Primeramente, los participantes fueron informados de la naturaleza y condiciones del experimento, así como del compromiso de confidencialidad por parte del investigador quedando cada individuo con una copia.

En segundo lugar, se les pidió una autorización firmada para utilizar los datos personales que aportaban.

Por último, los participantes cumplimentaron un cuestionario sobre aspectos personales que podrían ser utilizados como criterios de exclusión o para aclarar algún aspecto no cubierto por la muestra de cortisol o por el test.

Los siguientes registros se realizaron de forma conjunta a todos los individuos en un mismo día, hora y lugar, para evitar la posible variación hormonal debida al ritmo circadiano.

\section{Variables psicológicas}

Para la medida de las características conductuales se utilizó el Cuestionario de Personalidad CEP, de Pinillos ${ }^{28}$. Este cuestionario recoge distintos aspectos como: estabilidad emocional (C), extraversión (E), paranoidismo $(\mathrm{P})$ y sinceridad $(\mathrm{S})$.

La variable escala de control (C) refleja una emotividad interna, casi endógena, que escapa al propio control del individuo. A mayores valores en esta escala, mayor control emocional

La variable extraversión (E) recoge optimismo y sociabilidad, a la vez que gusto por las relaciones sociales.

El factor paranoidismo (P) muestra agresividad, suspicacia y sentimientos negativos respecto a las intenciones ajenas en aquellos individuos con mayores tasas.

Por último, la información aportada por la escala sinceridad (S) no será considerada útil para el experimento.

La corrección de los tests se hizo de forma manual según instrucciones del propio test. 


\section{Variables físicas}

Para la toma de muestras de hormona cortisol se utilizaron recipientes Salivette ${ }^{\circledR}$ de Sarstedt.

Previamente al experimento se pidió a los sujetos que no consumieran alimentos sólidos y líquidos 30 minutos antes de la prueba, tal y como aconseja el fabricante. Para asegurar este punto, la toma de muestras de cortisol se hizo tras la explicación y realización del test de personalidad, que llevó cerca de 1 hora. Se permitió beber agua a las participantes que lo necesitaron.

Una vez tomadas las muestras, la custodia de frío se cumplió también según instrucciones del fabricante, mediante congelación hasta su análisis en laboratorio.

El análisis de la hormona se realizó mediante el kit DEMEDITEC Cortisol ELISA en los laboratorios del Departamento de Psicobiología de la UNED en Madrid.

Para el análisis y cruce de los datos aportados por los registros psicológicos y fisiológicos se utilizó el programa informático SPSSPC versión 15 para Windows con licencia de uso para la Universidad Complutense de Madrid.

Se utilizarán pruebas paramétricas como el test de Levene para la homogeneidad de las varianzas, el test de Kolmogorov-Smirnov para la normalidad, el estadístico de Welch para la igualdad de medias y el test de correlación de Pearson. Para evitar los valores extremos de los grupos experimentales tan reducidos se emplearán las pruebas no paramétricas U de Mann-Whitney y test de correlación de Spearman.

\section{RESULTADOS}

Mediante la prueba $\mathrm{T}$ para dos muestras independientes, del paquete SPSSPC versión 15, comparamos primeramente las medias de cortisol, control, extraversión, paranoidismo y edad entre ambos grupos (primera y segunda hipótesis) encontrando diferencias importantes en las variables control emocional y paranoidismo, aunque las diferencias no son significativas en la hormona cortisol. A continuación mostramos en la figura 1 los resultados encontrados en los factores psicológicos.

Según muestra un primer análisis de los datos utilizando el test de Levene, las varianzas no son homogéneas (homocedasticidad), ni

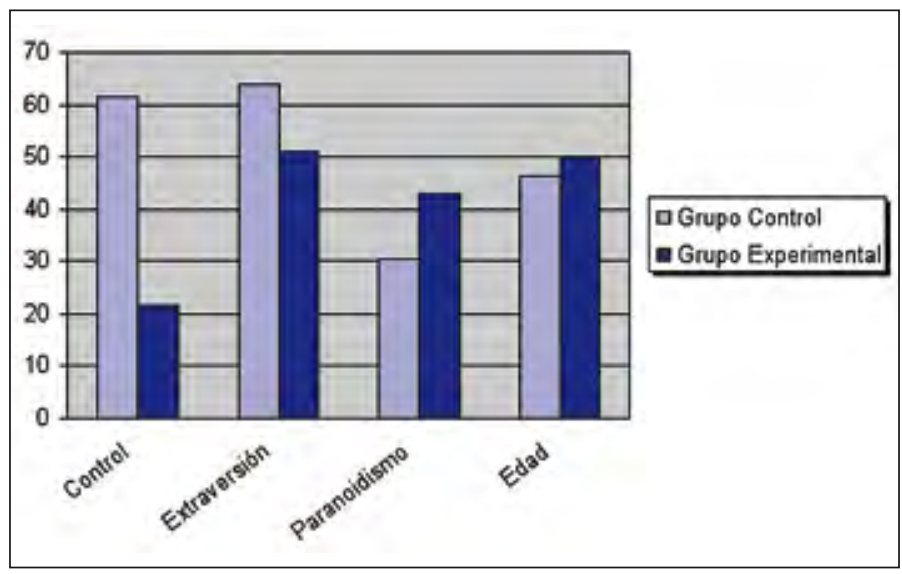

Figura 1. Los niveles de Control Emocional del grupo experimental resultan hasta 3 veces inferiores al grupo control, al igual que son evidentes las diferencias en el factor Paranoidismo. en paranoidismo ni en cortisol. Por tanto no se cumplen los requisitos de la prueba $\mathrm{T}$ de homocedasticidad y de normalidad para el cortisol en el test de Kolmogorov-Smirnov.

Encontramos que la diferencia de medias resulta también significativa, por lo que continuaremos buscando una relación entre las variables.

Puesto que no se cumplen los supuestos de normalidad y homocedasticidad, realizamos el análisis no paramétrico mediante la prueba U de Mann-Whitney.

Esta vez sí encontramos diferencias significativas de los niveles de cortisol $(0,048)$ y control 0,00$)$, entre ambos grupos. El resto, paranoidismo y extraversión no resultan significativos.

Realizamos la comprobación de correlaciones (tercera hipótesis), sin encontrar a priori significación en la correlación de Pearson. Por ello, elegimos las pruebas no paramétricas para evitar los valores extremos debidos al número escaso de sujetos y a la variabilidad de estos. Esta vez, el test de correlación de Spearman sí refleja valores significativos: correlación entre cortisol y grupo; correlación inversa entre control y paranoidismo (a mayor control, menor paranoidismo); correlación entre control y grupo; correlación inversa entre extraversión y paranoidismo (a mayor extraversión, menor paranoidismo).

\section{DISCUSIÓN}

Según veíamos en los resultados, encontramos diferencias significativas en algunas de las variables estudiadas.

La primera hipótesis buscaba relacionar la FM con un patrón específico de personalidad. La gran significatividad encontrada en la correlación entre los niveles de control emocional y grupo respalda esta hipótesis, puesto que el grupo con FM refleja unos niveles muy inferiores de Control Emocional con respecto al grupo libre de la enfermedad. Estos datos respaldan otras investigaciones que reclaman un perfil psicológico específico de las alteraciones relacionadas con el dolor, así como unas respuestas conductuales distintivas ${ }^{16}$.

Tal y como describe el manual del cuestionario CEP de Pini$1 \operatorname{los}^{28}$, la variable escala de control (C) es un concepto equivalente

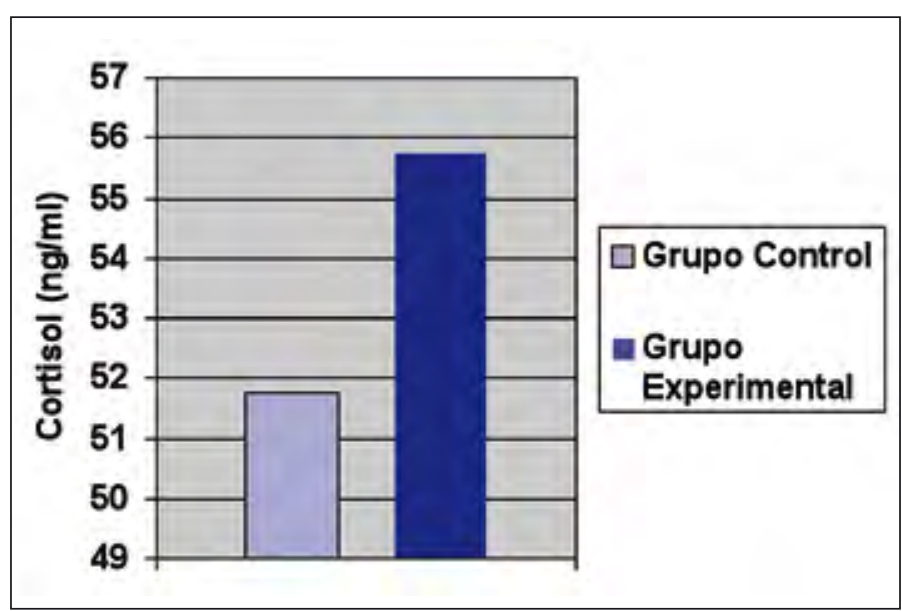

Figura 2. Mayor concentración de cortisol en los individuos con FM con respecto al grupo control. Sin embargo estas diferencias no son significativas a priori y requerirán un análisis estadístico más profundo. 
al de estabilidad emocional. De aquí, que este parámetro que hemos medido se pueda asemejar y apoye al de labilidad emocional o Neuroticismo descritos por Eysenck en su teoría de la personalidad ${ }^{17}$. Así como ocurre con el exceso de labilidad, la falta de control emocional produce, a nivel psicológico, una menor estabilidad personal en los sujetos con FM la cual -a su vez- desembocará en una activación fisiológica (arousal) más o menos alta y permanente en los individuos. Como consecuencia de lo anterior, se producirá una mayor sensibilidad hacia los estímulos físicos y psicológicos procedentes del exterior y las consiguientes reacciones conductuales -en algunos casos aparentemente exageradas-de alarma, lucha o huida.

Se entiende, entonces, que las capacidades afectivas de las personas con FM puedan quedar agotadas, incrementando así el grado de correlaciones negativas entre estados positivos y negativos, tal y como señalan Zautra y col $^{29}$. Estos sujetos pueden encontrar más difícil realizar respuestas de adaptación conductual positiva ante circunstancias adversas o traumáticas si la fuerza del afecto negativo compromete sus recursos de afectos positivos. Con el paso del tiempo, la incapacidad de mantener la homeostasis emocional en momentos de estrés, puede generar a los pacientes de FM una percepción de los eventos como más estresantes o desequilibrantes, resultando en una perpetuación de este ciclo. Así, dicho estudio también validaría el resultado encontrado en nuestro experimento en el sentido de que el control emocional, bien como causa o bien como consecuencia, se vería afectado por el dolor crónico mantenido en el tiempo.

Desde este modelo de emociones y estrés propuesto, se puede entender que las tasas de depresión habitualmente encontradas en pacientes con FM podrían ser explicadas, sobretodo, por el sentimiento de indefensión aprendida y por la ansiedad que experimentan los pacientes debido al desconocimiento de la etiología de la FM, tal y como proponen algunos autores ${ }^{15}$. Además, se podría añadir que la incertidumbre y la falta de control percibida acerca de las reacciones del propio cuerpo ante los diversos estresores, colaboran enormemente en incrementar esta sensación de depresión/ansiedad, que, en suma, forman el factor control emocional (C) reflejado en el test.

Estas conclusiones encuentran apoyos en estudios que utilizan diferentes tests, como el Minnesota Multiphasic Personality Inventory 2 (MMPI-2). Blasco encuentra dos perfiles psicológicos diferentes que explicarían la enfermedad: un perfil A (tipo DC en el MMPI-2), relacionado con las enfermedades crónicas y caracterizado por la preocupación de los síntomas somáticos, ansiedad frente a la enfermedad, propensión a desarrollar síntomas físicos frente al estrés, y un perfil B (tipo DP según el MMPI-2) determinado sobretodo por bajos recursos personales frente a las situaciones de estrés y miedo a los conflictos ${ }^{30}$. Se encuentran resultados similares en otros estudios que utilizan este test.

Al realizar el test de Levene, se observó una significación en las diferencias en el factor edad. Pese a que la diferencia de las medias de edad resulta significativa a nivel estadístico, no lo es tanto a nivel biológico, puesto que hablamos de unas medias que rondan entre los 46 y los 49 años de edad de los sujetos. Los estudios científicos que encuentran diferencias significativas entre cortisol y edad utilizan rangos de edades con diferencias mucho mayores entre sí (grupos de media de 24,4 años frente a medias de 81,6 años $)^{31,32}$. Por ello, pese a la significación de las diferencias de edad, no tomaremos este dato como influyente en los resultados.
La segunda hipótesis de este estudio trataba de averiguar si las personas con FM muestran niveles de cortisol superiores al grupo experimental. Los resultados encontrados señalan que el grupo con FM efectivamente muestra una media de cortisol superior frente al grupo control $(55,75 \mathrm{ng} / \mathrm{ml}$ frente a $51,76 \mathrm{ng} / \mathrm{ml})$, y que las correlaciones entre las variables cortisol y grupo poseen diferencias estadísticamente significativas, con lo cual creemos que la hipótesis quedaría validada. Estos datos se apoyan en estudios como el de Melzack $^{11}$, quien reconoció la relación existente entre niveles altos de cortisol y la existencia de algunos dolores crónicos debidos al estrés. Desde esta perspectiva, los niveles basales de cortisol aumentarían en el grupo experimental fruto de la arousal que comentábamos anteriormente, y estos permanecerán constantemente elevados en mayor medida si los comparamos con los sujetos libres de FM, tal y como demuestra el análisis de los datos.

Otros autores que también buscaron evidencias de esta relación, encontraron una hiperactividad del Sistema Nervioso simpático en pacientes con FM durante el período nocturno que además coincidía con un incremento de episodios de despertar nocturnos y arousal elevado. Las diferencias en el cortisol basal recogido en nuestro experimento reflejarían pues, la hiperactividad nocturna que apuntaba el citado estudio ${ }^{33}$.

McLean y col encuentran una fuerte relación entre los niveles de cortisol matutino y los síntomas dolorosos de los pacientes con FM, aunque no encuentran estos niveles de cortisol en los registros posteriores que hizo a lo largo del día ${ }^{34}$.

Bengtsson ${ }^{35}$ encontró una reducción del número de puntos gatillo y puntos sensibles en pacientes con FM al bloquear el Sistema Nervioso Simpático con bupivacaína. La conclusión fue que la actividad de dicho sistema podía contribuir en algunos individuos a la patogénesis de la FM primaria.

Por último, Martínez-Lavín propone la hiperactividad del sistema adrenérgico como la explicación de varios de los síntomas de la FM. Así, esta hiperactividad establecería conexiones anormales entre el sistema nervioso simpático y las fibras nociceptivas ${ }^{36}$.

Sin embargo, los resultados encontrados en este experimento se muestran en contradicción con los aportados por otros autores que detectan niveles inferiores de cortisol tanto en orina como en sali$\mathrm{va}^{18,19,25}$. Incluso en algunos casos se ha encontrado una evolución a lo largo del día en los niveles de cortisol y, mientras los niveles hormonales obtenidos en muestras de orina a primera hora de la mañana son sensiblemente inferiores en comparación con el grupo control, al final de la tarde los niveles de cortisol se incrementan por encima incluso de los niveles del grupo control ${ }^{20}$. Sin embargo, y en frontal oposición a los datos anteriormente señalados, el mismo autor encuentra en su investigación que los niveles de cortisol plasmático por la mañana son similares en ambos grupos $(276 \mathrm{nmol} / \mathrm{L}$ frente a $283 \mathrm{nmol} / \mathrm{L}$ ). Vemos que pese a que los datos de las investigaciones expresan de forma relativamente habitual la disminución de los niveles de cortisol, no hay una postura unánime, por lo que creemos que sería deseable incrementar el número de investigaciones al respecto.

La ausencia de un registro que incluya medidas del cortisol matutino y vespertino en el experimento que hemos realizado, impide contrastar los datos de las otras investigaciones citadas en los que se observan conclusiones diferentes. Pese a que los resultados del presente estudio han sido fruto de una metodología y análisis exhaustivos, sería conveniente tomar un mayor número de muestras para clarificar esta aparente falta de consenso. 
En cualquier caso, existen algunas evidencias de que las alteraciones del estado del ánimo correlacionan con niveles altos de cortisol salivar. Un estudio que trabaja en este sentido es el llevado a cabo por Lieb ${ }^{37}$. En él, pacientes con Trastorno de Personalidad Límite (TPL) muestran un incremento de cortisol salivar diurno, tanto por la mañana como durante todo el día. El TPL se caracteriza por un patrón de inestabilidad en la regulación afectiva, en el control de los impulsos o en las relaciones interpersonales, entre otros, conceptos todos ellos muy relacionados con el de la variable control emocional (C) medida en el test utilizado en el experimento. Aunque ambas enfermedades no sean comparables en su totalidad, las semejanzas encontradas en los síntomas emocionales y en los niveles altos de cortisol, podrían sugerir que comparten algunas bases fisiológicas aún no demostradas, pero que con una investigación escrupulosa podrían ser determinadas.

La tercera hipótesis intentaba demostrar la relación inversa entre cortisol y control emocional. Los datos a este respecto resultan paradójicos. A pesar de que tanto el cortisol como la escala de control (C) correlacionan por separado con la variable grupo - evidenciando así las diferencias ya apuntadas entre la condición FM y la condición de sujetos sanos- no se encuentran resultados suficientemente significativos para considerar esta relación inversa entre cortisol y control emocional. La explicación de este desajuste puede ser debida a que la variable control emocional no interaccione en todos los casos con la hormona corticoide y que, por tanto, el factor psicológico control emocional se vea alterado por otras variables físicas o psicológicas distintas.

De hecho, los resultados del cuestionario señalan que control emocional y paranoidismo sí correlacionan inversamente, por lo que esta relación resultaría más concluyente que la expuesta en la tercera hipótesis. La escala de paranoidismo $(\mathrm{P})$ refleja, según su autor, agresividad, suspicacia y tenacidad rígida, además de una concepción negativa de las intenciones ajenas. Los conflictos sociales pueden ser consecuencia del grado de vulnerabilidad al estrés que sufren los pacientes de FM. Davis y col. encontraron evidencias de que las respuestas sociales se acortaban en estos pacientes y que incluso se disminuían los intercambios positivos en comparación con un grupo control. Los autores atribuían esta conducta antisocial a una incapacidad por parte de los afectados de prevenir los episodios de dolor ${ }^{38}$. Para Pérez-Pareja, la ansiedad en personas con FM está relacionada con las experiencias de dolor ${ }^{15}$. Se entiende, por tanto, que aquellas personas con menor control emocional juzguen las situaciones o personas que le produzcan ansiedad como más agresivas o negativas, reflejando en el test una puntuación mayor en esta escala. Un estudio destaca la importante relación entre la focalización en lo somático, las emociones negativas y la experiencia dolorosa que podría relacionarse con esta variable de personalidad. Según los autores, el dolor será mayor en las personas que centran su atención en las sensaciones somáticas, a la vez que desarrollarán unas emociones negativas de formas más habitual. Así, se entiende que los afectados de FM, muy limitados físicamente y obligados a focalizar su atención en el dolor, muestren una actitud más clara de rechazo o de prevención frente a lo ajeno o frente a situaciones que escapan a su control ${ }^{39}$.

La última correlación que encontramos en los resultados es la existente entre paranoidismo (P) y extraversión (E), que lo hacen inversamente. Esta escala de extraversión es un factor que recoge tendencias opuestas a la de paranoidismo en el sentido de que refleja un factor psicológico apoyado en una fisiología libre de ansiedad, angustia o desconfianza. Así, resulta lógico entender que los valores altos de paranoidismo vayan en detrimento de la variable extroversión. En este caso, los valores del grupo con FM, al estar más condicionados por la tendencia de su sistema nervioso hacia la experiencia de dolor, muestran menor predisposición a la extraversión y a la sociabilidad que el grupo control.

Volviendo de nuevo a la teoría de la personalidad de Eysenck -el test que hemos utilizado toma a dicho autor como referencia para el estudio de la conducta- podemos suscribir sus palabras en el sentido de que, efectivamente, la extraversión está relacionada con un nivel bajo de activación cortical crónica. Como se observa en los resultados, pese a no haber una correlación significativa entre ambas variables, el grupo control obtiene unas puntuaciones más altas en extraversión que el grupo experimental, así como unas puntuaciones más bajas en cortisol. No podemos tomar estos resultados como firmes, pero una primera comparación llama la atención sobre este hecho que, en cualquier caso, necesitaría una experimentación más exhaustiva.

Por último, la falta de normalidad (la variable dependiente se distribuye normalmente en las poblaciones muestreadas) y homocedasticidad (las poblaciones muestreadas poseen la misma varianza) encontradas al comienzo del análisis de los resultados y que fueron suplidas por el uso de análisis no paramétricos, sugieren que es deseable una investigación más amplia en cuanto a número de sujetos, además de una recogida de muestras matutinas y vespertinas de cortisol.

\section{CONCLUSIONES}

Retomando las hipótesis planteadas al inicio podemos decir que:

El factor control emocional (C) es un elemento diferenciador de los afectados por FM con respecto a la población sana. A nivel psicológico la variable control (C) muestra una significación estadística sólida que refleja claramente el menor control emocional de sujetos con FM respecto a sujetos sanos.

La variable cortisol también es un factor diferenciador de la condición grupo FM. Sin embargo, aunque apoya la hipótesis inicial propuesta de que las personas afectadas por FM muestran mayores tasas en sujetos con FM, lo hace con una significación estadística menor que la variable control emocional (C) y en aparente contradicción con estudios anteriores.

No se encuentra una correlación significativa entre los niveles de cortisol elevados y rasgos de personalidad de bajo control emocional. Entendemos que este hecho pueda deberse a que los niveles de cortisol sean alterados por factores diferentes del propuesto control emocional y viceversa. En cualquier caso, las otras correlaciones encontradas (control y paranoidismo, control y extraversión, y paranoidismo y extraversión) pueden resultar de gran interés en el tratamiento y futuras investigaciones sobre la FM.

\section{BIBLIOGRAFÍA}

1. Smythe H. \& Moldofsky H. (1978). Two contributions to understanding the «fibrositis» syndrome. Bulletin of Rheumatic Disease, 28(1), 928-931.

2. Wolfe F, Ross K, Anderson J, Russell IJ, \& Hebert L. (1995). The prevalence and characteristics of fibromyalgia in the general population. Arthr Rheum, 38(1), 19-28. 


\section{Estabilidad emocional y cortisol como factores diferenciadores de la fibromialgia}

3. Wolfe F., Smythe H.A., Yunus M.B., Bennett R.M., Bombardier C., Goldenberg D.L., Tugwell P., Campbell S.M., Abeles M., Clark P., Fam A.G., Farber S.J., Fiechtner J.J., Franklin C.M., Gatter R.A., Hamaty D., Lessard J., Lichtbroun A.S., Masi A.T., McCain G.A., Reynolds W.J., Romano T.J., Russell I.J., \& Sheon R.P (1990). The American College of Rheumatology 1990 criteria for the classification of fibromyalgia: Report of the multicenter criteria committee. Arthritis and Rheumatism 33(2), 160-177.

4. Yunus M.B., Masi A.T. \& Aldag J.C. (1989). A controlled study of primary fibromyalgia syndrome: clinical features and association with other functional syndromes. J Rheumatol Suppl.19, 62-71.

5. Robbins J.M., Kirmayer L.J. \& Hemami S. (1997). Latent variable models of functional somatic distress. Journal of Nervous and Mental Disease 185(10), 606-615.

6. Komaki G., Moriguchi Y., Ando T., Yoshiuchi K.\& Nakao M. (2009). Prospects of Psychosomatic Medicine; BioPsychoSocial Medicine, 3,1.

7. Selye, H. (1946). The general adaptation syndrome and the diseases of adaptation. Journal of Clinical Endocrinology, 6, 117-230.

8. Ursin H. \& Eriksen H. (2001) Sensitization, subjective health complaints and sustained arousal. Ann NY Acad Sci 933, 119-129.

9. Martin, A.L., McGrath P.A., Brown S.C. \& Katz J.(2007, Winter). Anxiety sensitivity, fear of pain and pain-related disability in children and adolescents with chronic pain. Pain Res Manage, 12(4), 267-72.

10. Dersh J, Polantin PB, Gatchel RJ. (2002) Chronic pain and psychopathology: research finding and theoretical considerations. Psychosom Med, 64, 773-786.

11. Melzack R. (2000) Del umbral a la neuromatriz. Rev Soc Esp Dolor, 7, 149-156.

12. Hudson J.I., Hudson M.S., Pliner L.F., Goldenberg D.L. \& Pope H.G. Jr (1985) Fibromyalgia and major affective disorder: A controlled phenomenology and family history study. American Journal of Psychiatry, 142(4), 441-446.

13. Krag N., Norregaard, Larsen J.K. \& Danneskiold-Samsoe B. (1994). A blinded, controlled evaluation of anxiety and depressive symptoms in patients with fibromyalgia, as measured by standardized psychometric interview scales. Acta Psychiatrica Scandinavica 89 (6), 370-375.

14. Walker E.A., Keegan D., Gardner G., Sullivan M., Katon W.J. \& Bernstein D. (1997). Psycosocial factors in fibro0myalgia compared with rheumatoid arthritis I. Psychiatric diagnoses and functional disability. Psychosomatic Medicine 59(6), 565-571.

15. Pérez-Pareja, J; Borrás, C; Palmer, A; Molina F \& Gonzalvo J. (2004). FM y emociones negativas. Psicothema, 16 (3), 415-420.

16. Ramírez, C.; López, A.E. \& Esteve, R. (2004). Personality characteristics as differential variables of the pain experience. Journal of Behavioral Medicine, 27 147-165.

17. Eysenck H.J. (1967). Biological basis of personality. Springfield, II. Charles Thomas.

18. McCain G.A. \& Tilbe K.S. (1989). Diurnal hormone variation in fibromyalgia syndrome: a comparison with rheumatoid arthritis. J.Rheumatol.16, 154-157.

19. Griep E.N., Boersma J.W. \& de Kloet E.R. (1993). Altered reactivity of the hypothalamic-pituitary-adrenal axis in the primary fibromyalgia syndrome. Journal of Rheumatology, 20(3), 469-474.

20. Crofford L.J., Pillemer S.R., Kalogeras K.T., Cash L.M., Michelson D., Kling M.A., Sternberg E.M., Gold P.W., Chrousos G.P., Wilder R.L. (1994). Hypothalamic-pituitary-adrenal axis perturbations in patients with fibromyalgia. Arthritis and Rheumatism, 37(11), 1583-1592.

21. Crofford L.J., Young E.A., Egleberg N.C., Korszun A., Brucksch C.B., McClure L.A. et al.. Basal circadian and pulsatile ACTH and cortisol secretion in patients with fibromyalgia and/or chronic fatigue syndrome. Brain Behav Immun. 2004, $18,314-325$.
22. Lentjes E.G.W.M., Griep E.N., Boersma J.W., Romijn F.P.T.H.M. \& de Kloet E.R (1997). Glucocorticoid receptors, FM and low back pain. Psychoneuroendocrinology 22(8), 603-614.

23. Catley D., Kaell A.T., Kirschbaum C. \& Stone A.A. (2000). A naturalistic evaluation of cortisol secretion in persons with fibromyalgia and rheumatoid arthritis. Arthritis Care and Research, 13 (1), 51-61.

24. Crofford L.J., Young E.A., Egleberg N.C., Korszun A., Brucksch C.B., McClure L.A., Brown M.B. \& Demitrack M.A. (2004). Basal circadian and pulsatile $\mathrm{ACTH}$ and cortisol secretion in patients with fibromyalgia and/or chronic fatigue syndrome. Brain, Behavior, and Immunity, 18, 314-325.

25. Macedo J.A., Hesse J., Turner J.D., Meyer J., Hellhammer D.H. \& Muller C.P (2008). Glucocorticoid sensitivity in fibromyalgia patients: Decreased expression of corticosteroid receptors and glucocorticoid-induced leucine zipper. Psychoneuroendocrinology 33, 799-809.

26. Landis C.A., Lentz M.J., Rothermel J., Riffle S.C., Chapman D., Buchwald D. \& Shaver J.L.F. Decreased Nocturnal Levels of Prolactin and Growth Hormone in Women with Fibromyalgia. The Journal of Clinical Endocrinology \& Metabolism 86 (4).

27. Gur A., Cevik R., Nas K., Colpan L \& Sarac S. (2004). Cortisol and hypothalamic-pituitary-gonadal axis hormones in follicular-phase women with fibromyalgia and chronic fatigue syndrome and effect of depressive symptoms on these hormones. Arthritis Research \& Therapy, 6(3).

28. Pinillos J.L. (2003). Cuestionario de personalidad CEP (9th. ed). Madrid: TEA Ediciones S.A

29. Zautra A.J., Berkhof J. \& Nicolson N.A (2002). Changes in affect interrelations as a function of stressful events. Cognition and Emotion, 16, 309-18.

30. Blasco L., Mallo M., Mencía A., Franch J., Casaus P., Peña J., et al. (2006). Clinical profiles in fibromyalgia patients of the community mental health center: a predictive index of psychopathological severity. ActasEspPsiquiatr. 34, 112-22.

31. Born J., Ditschuneit I., Schreiber M., Dodt C. \& Fehm H.L. (1995). Effects of age and gender on pituitary-adrenocortical responsiveness in humans. Eur J Endocrinol 132, 705-11.

32. Ferrari E., Cravello L., Muzzoni B., Casarotti D., Paltro M., Solerte S.B., Fioravanti M., Cuzzoni G., Pontiggia B. \& Magri F. (2001). Age-related changes of the hypothalamic-pituitary-adrenal axis: pathophysiological correlates. European Journal of Endocrinology, 144, 319-329.

33. Kooh M., Martínez-Lavín M., Meza S., Martín-del-Campo A., Hermosillo A.C. Pineda C., Naval A., Amigo M. ${ }^{a}$ \& Drucker-Colin R. (2003). Concurrent heart rate variability and polysomnography analyses in fibromyalgia patients. Clin Exp Rheumatol, 21, 529-530.

34. McLean S.A., Williams D.A., Harris R.E., Kop W.J., Groner K.H., Ambrose K., Lyden A.K., Gracely R.H, Crofford L.J., Geisser M.E., Sen A., Biswas P., \& Clauw D.J. (2005). Momentary relationship between cortisol secretion and symptoms in patients with Fibromyalgia. Arthritis and rheumatism 52, (11), 3660-3669.

35. Bengtsson A., Bengtsson M. (1988). Regional sympathetic blockade in primary fibromyalgia. Pain, 33, 161-167.

36. Martínez-Lavín M. (2007). Stress, the stress response system, and fibromyalgia. Arthritis Research \& Therapy, 9(4), 216.

37. Lieb K., Rexhausen J.E., Kahl K.G., Schweiger U., Philipsen A., Hellhammer D. H. \& Bous M. Increased diurnal salivary cortisol in women with borderline personality disorder. Journal of Psychiatric Research, 38 (6), 559-565.

38. Davis M.C., Zautra A.J., Reich J.W. Vulnerability to stress among women in chronic pain from fibromyalgia and osteoarthritis. Ann Behav Med,;23, 215-26.

39. O’ Brien E.M., Atchison J.W., Gremillion H.A., Waxenberg L.B. \& Robinson M.E. (2008). Somatic focus/awareness: Relationship to negative affect and pain in chronic pain patients. Eur J Pain., 12(1), 104-115. 